\title{
William Morris et les arts du livre
}

William Morris and the Arts of the Book

\section{Antoine Capet}

\section{CpenEdition}

\section{Journals}

Édition électronique

URL : http://journals.openedition.org/rfcb/3314

DOI : $10.4000 /$ rfcb.3314

ISSN : 2429-4373

Éditeur

CRECIB - Centre de recherche et d'études en civilisation britannique

\section{Édition imprimée}

Date de publication : 1 septembre 2004

ISBN : 2-911580-19-2

ISSN : 0248-9015

\section{Référence électronique}

Antoine Capet, «William Morris et les arts du livre », Revue Française de Civilisation Britannique [En ligne], XIII-1 | 2004, mis en ligne le 01 septembre 2004, consulté le 07 octobre 2019. URL : http:// journals.openedition.org/rfcb/3314; DOI : 10.4000/rfcb.3314

Ce document a été généré automatiquement le 7 octobre 2019.

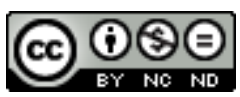

Revue française de civilisation britannique est mis à disposition selon les termes de la licence Creative Commons Attribution - Pas d'Utilisation Commerciale - Pas de Modification 4.0 International. 


\section{William Morris et les arts du livre}

William Morris and the Arts of the Book

\section{Antoine Capet}

Frontispice de l'édition Kelmscott Press de News from Nowhere (1892).

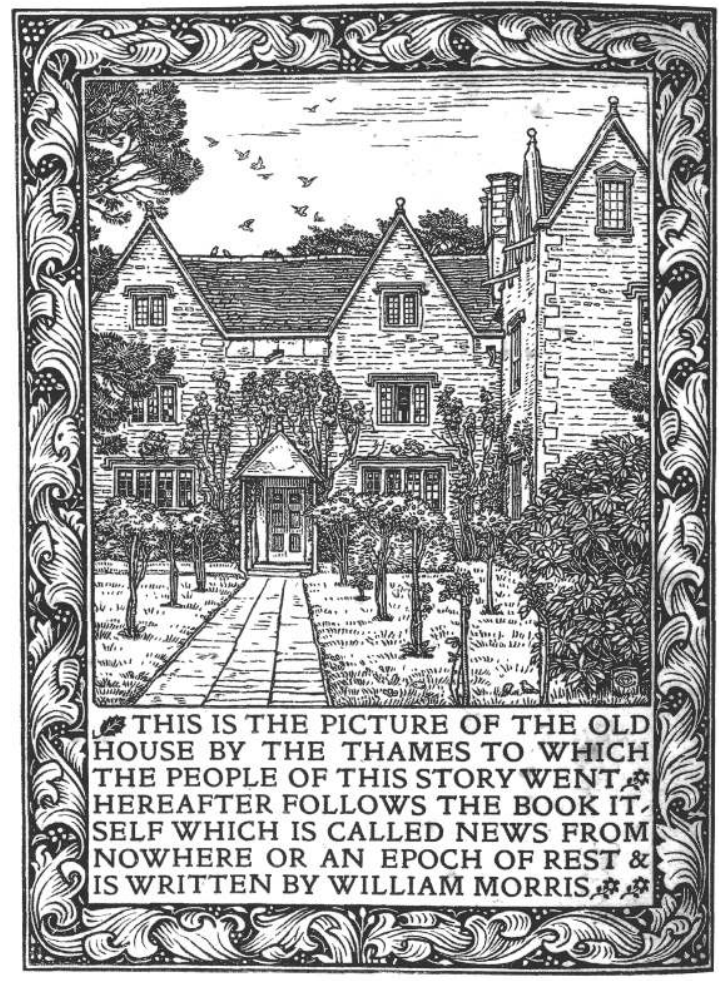

Le texte est en Golden Type

On connaît la quête de Richard Wagner (1813-1883), qui cherche à atteindre le Gesamtkunstwerk, l'œuvre d'art totale, à travers ses opéras ${ }^{1}$. Toutes proportions gardées, il semble que ce soit une quête de même nature qui occupe William Morris (1834-1896) dans les dernières années de sa vie, non pas à travers l'opéra, bien sûr, mais à travers le 
livre $^{2}$. Isolde Karen Herbert explique magnifiquement cette démarche globalisante, ainsi que son objet :

Morris believed that 'history has become a book from which the pictures have been torn'; ${ }^{3}$ his remedy was to recover (in both senses of the word) that book's material structure, illustrations, and narrative - forms which he hoped would foster the desire for the rebirth of art. ${ }^{4}$

2 Il existe au moins deux grandes catégories d'amoureux des livres : ceux qui en dévorent le contenu sans porter le moindre intérêt à leur aspect matériel et ceux pour qui le « plaisir du texte " est gâché lorsque l'œil déplore la piètre qualité du support. Est-il besoin de préciser que William Morris se rangeait dans la seconde catégorie? Nous possédons à cet égard plusieurs indications, à commencer par le très instructif entretien avec le journaliste de la Pall Mall Gazette aux premiers temps de la Kelmscott Press :

'If larger and better type were generally adopted, there would be an initial expense for the type, and that would be comparatively large; but think of the benefit that would be conferred on the public! No more of the horrible type in cheap editions which the millions now have to read, and by which they spoil their eyesight.'

'But, Mr. Morris, is it not better to give them books with small type, which they can buy cheap, than to prevent them from reading at all, which would the case if there were no small type and consequently cheap editions?'

3 À quoi Morris répond, convaincu que l'avènement du socialisme permettra de résoudre la contradiction actuelle entre l'élitisme du plaisir esthétique réservé aux riches et la nécessité de permettre au plus grand nombre d'accéder d'une autre façon que la possession capitaliste à ce plaisir :

'Well, yes, that is where the difficulty lies. You see,' Mr. Morris went on, with a sudden smile flitting over his face as he turned to the old, old story, 'you see if we were all Socialists things would be different. We should have a public library at each street corner, where everybody might see and read all the best books, printed in the best and most beautiful type. I should not then have to buy all these old books, but they would be common property, and I could go and look at them whenever I wanted them, as would everybody else. Now I have to go to the British Museum, which is an excellent institution, but it is not enough. I want these books close at hand, and frequently, and therefore I must buy them. It is the same with everybody else, and if they have not money enough to buy them they must go without. Socialism would alter all that.'

'It would give us a hundred British Museums instead of the one, ${ }^{5}$ you mean, Mr. Morris,'

'Just so.'

Par ailleurs, même si le témoignage de May Morris n'a souvent que la valeur d'une hagiographie filiale, on ne peut tenir pour quantité négligeable ce qu'elle rapporte sur sa "passion » de bibliophile : 'It was a great passion, not exactly the collector's passion... He loved his books as a craftsman, as a poet, as a romancist: with a threefold affection and a threefold pleasure'?

Jugement corroboré par celui du journaliste anonyme de la Pall Mall Gazette, qui relate 'the evident delight of a true lover of old books, to whom his treasures are a never-ceasing source of enjoyment." Peterson ira plus loin, parlant de 'Morris's... almost sensual pleasure in the ownership of beautiful books.' D'autres plus loin encore : 'A satisfying book evoked from Morris a positively erotic response'. ${ }^{10}$

6 Grâce à son aisance matérielle, William Morris constitue tout au long de sa vie une remarquable collection de manuscrits médiévaux enluminés ${ }^{11}$ et d'incunables ${ }^{12}$. En 
général, les bibliophiles s'arrêtent là. Mais William Morris va deux étapes plus loin. Il donne des conférences et il publie des écrits théoriques sur ce qui fait selon lui la qualité esthétique d'un livre. Bien plus, contredisant une fois de plus la cruelle formule : 'Those who can, do - those who cannot, teach', il se lance en 1891 dans la fabrication de livres en fondant la Kelmscott Press.

\section{Le livre et l'architecture}

7 Une conférence de 1893 intitulée The Ideal Book ${ }^{13}$ recense les qualités principales que William Morris attend d'un "beau livre ", mais d'autres textes donnent encore plus de détails sur les exigences à respecter et les techniques à utiliser. D'abord, on peut faire un beau livre quel que soit le sujet car la réussite esthétique de la page imprimée dépend avant tout de l'harmonie entre la typographie, l'ornementation, les illustrations, la mise en page et la nature du texte :

A work on differential calculus, a medical work, a dictionary, a collection of a statesman's speeches, or a treatise on manures, such books, though they might be handsomely and well printed, would scarcely receive ornament with the same exuberance as a volume of lyrical poems, or a standard classic, or such like... Still, whatever the subject-matter of the book may be, and however bare it may be of decoration, it can still be a work of art, if the type be good and attention be paid to its general arrangement. ${ }^{14}$

Mais ce n'est là que l'idée générale, le principe directeur. William Morris revient sur chacun de ces points en détail, aussi bien dans cette conférence que dans une douzaine d'essais, de conférences et d'entretiens parus dans la presse sur le même sujet entre 1891 et sa mort, et que William S. Peterson a rassemblés dans son recueil publié en 1982 portant ce même titre de The Ideal Book. Susan Otis Thompson, de son côté, a eu l'idée de recenser tous les 'Statements on Book Design' qu'elle a pu trouver chez William Morris et de les classer selon le nombre de lignes qu'il consacre aux différents aspects du livre, ce qui devrait théoriquement indiquer l'importance qu'il leur accordait. La rubrique la plus fournie est celle des "Caractères». Vient ensuite la "Composition», divisée en " Espacement des lettres et des mots », "Interlignes ", " Marges ». Les autres rubriques portent sur le papier, la décoration et l'harmonie d'ensemble ; curieusement, la dernière et la moins fournie traite de l'analogie avec l'architecture ${ }^{15}$. Or, ce lien est fondamental de l'aveu même de William Morris, qui déclare :

If I were asked to say what is at once the most important production of Art and the thing most to be longed for, I should answer, A beautiful House; and if I were further asked to name the production next in importance and the next thing to be longed for, I should answer, A beautiful Book. To enjoy good houses and good books in self-respect and decent comfort, seems to me to be the pleasurable end towards which all societies of human beings ought now to struggle. ${ }^{16}$

En outre, il poursuit sa définition du " livre idéal » en le qualifiant de 'architecturally good' et en parlant de la conception du livre comme d'un 'architectural arrangement' pour terminer en disant que l'ornementation réussie 'must become architectural'; alors le livre 'may become a work of art second to none, save a fine building ${ }^{17}$ duly decorated, or a fine piece of literature..$^{18}$ ' 'est dans cette dernière phrase, semble-t-il, que William Morris révèle la véritable nature de sa bibliophilie : le livre, c'est la fusion des deux arts majeurs à ses yeux, l'architecture et la littérature. On comprend dès lors la passion qu'il met à étudier, puis à pratiquer, ce qu'on appelle les arts du livre et, connaissant son caractère, la profusion de sentences définitives et pas toujours heureuses sur les 
conditions que doit remplir le «beau livre». Deux exigences s'imposent immédiatement à lui : l'adoption d'une technique graphique propre à satisfaire sa quête du beau et le choix de matériaux en harmonie à la fois avec son goût et avec le respect de la meilleure tradition.

\section{La technique graphique}

Figure 1 : Incipit de l'édition Kelmscott Press de News from Nowhere (1892).

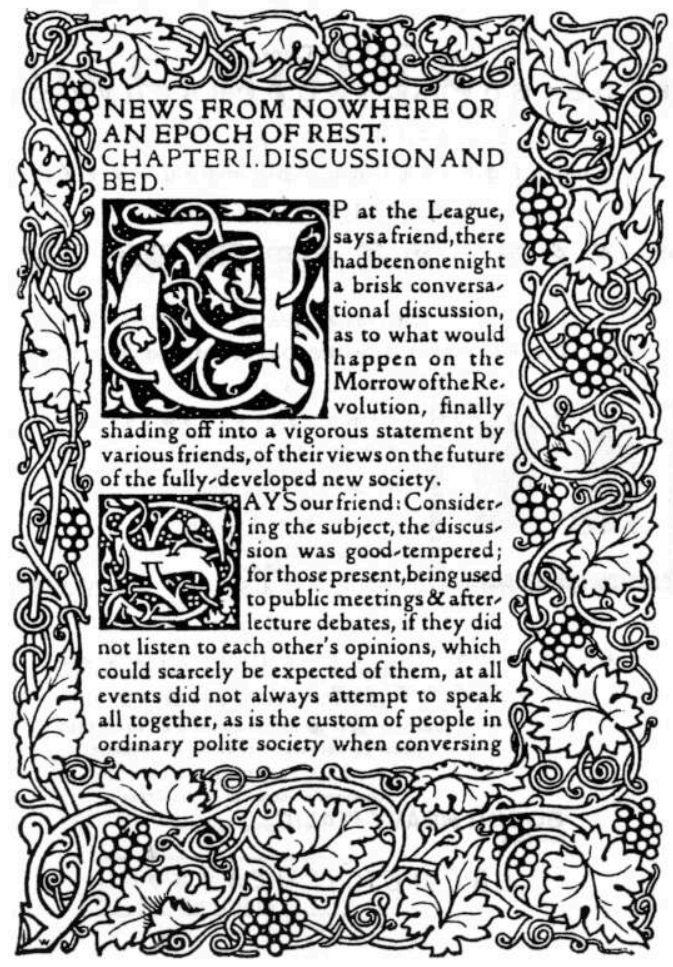

Imprimée en Golden Type : on notera le peu de différence entre les pleins et les déliés, entre la hauteur et la largeur des majuscules (notamment le $\mathrm{H}$ ), ainsi que la petite taille de leurs empattements. Remarquer également le peu de «blanc » qui reste sur la page, et la disproportion voulue entre la largeur des quatre bordures florales.

10 Homme de contradictions, Morris commence sa Note on his Aims in founding the Kelmscott Press en déclarant : 'I began printing books with the hope of producing some which would have a definitive claim to beauty, while at the same time they should be easy to read'. ${ }^{19}$ Cependant, dans son volume de la Kelmscott Press le plus "populaire" (avec tous les guillemets que l'on peut mettre ici à ce mot), le News from Nowhere de 1892, des groupes de mots comme 'Chapter I' ou 'Morrow of the Revolution', sans parler du $U$ enluminé suivi d'un $P$ en capitales un peu perdu à la première ligne, ne brillent pas par leur clarté, c'est le moins que l'on puisse dire (voir Fig. 1). 
Figure 2 : Giambattista Bodoni (1740-1813) : Manuale Tipografico (posthume, 1818).

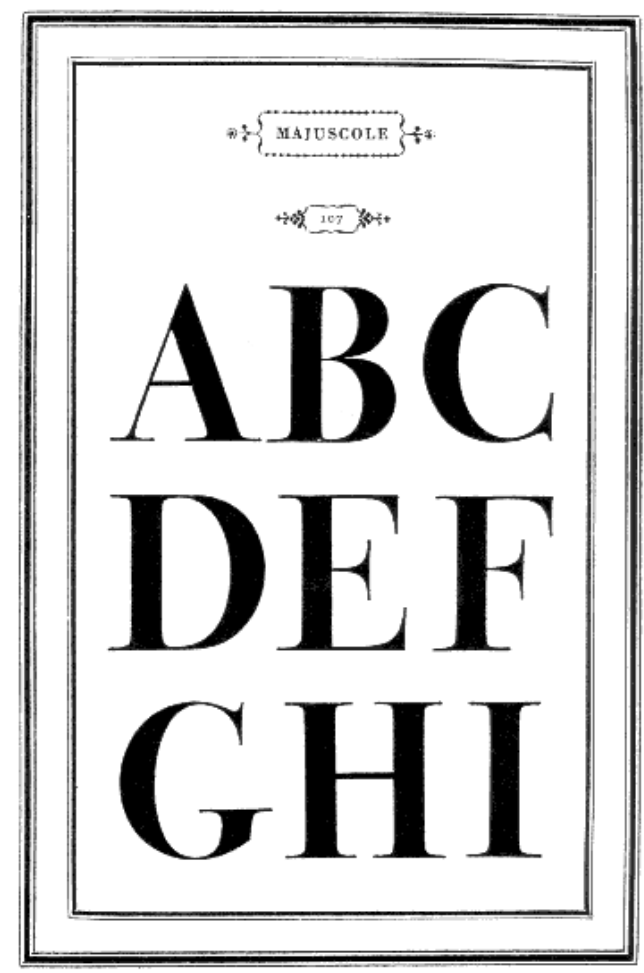

Page de majuscules romaines, montrant toutes les caractérisques que Morris abomine ('the most illegible type that was ever cut') : très fins empattements, contraste fortement marqué entre les pleins et les déliés, hauteur nettement supérieure à la largeur.

11 Par ailleurs, Morris juge 'positively ugly' les caractères de la fin du XVIII siècle que nous considérons aujourd'hui comme magnifiques, tels les Bodoni ou les Didot, qualifiés de 'gross and vulgar' au motif qu'ils sont 'dazzling and unpleasant to the eye owing to the clumsy thickening and vulgar thinning of the lines'. ${ }^{20}$ Confondant à dessein, comme à l'accoutumée ${ }^{21}$, l'esthétique et l'utilitaire, il va même jusqu'à écrire dans The Ideal Book que le Bodoni (voir Fig. 2) est 'the most illegible type that was ever cut', à cause de sa 'sweltering hideousness'. ${ }^{22}$ Nous avons là un exemple extrême des partis pris qu'il affectionnait, car son caractère dérivé du gothique utilisé pour son édition de Chaucer (1896) est parfois difficilement lisible dans les titres en capitales (voir Fig. 4: 'Here beginneth the tales...') et approche carrément du rébus dans les cartouches qui accompagnent les initiales (voir Fig. 5 : 'Almighty' difficilement décryptable pour un œil non initié et qui ne connaît pas le texte d'avance).

Il en va de même en matière de composition et de mise en page. Il soutient qu'avec des caractères bien choisis, 'the words may be set much closer together, without loss of clearness', et il estime dans le même paragraphe que 'the general solidity of a page is much to be sought for'. ${ }^{23}$ Cela implique pour lui que les intervalles entre les lignes doivent également être réduits le plus possible. Quant à la mise en page, c'est-à-dire l'emplacement du texte sur la feuille ou encore la dimension des plages blanches, elle obéit à des règles très strictes, elles aussi dérivées du Moyen Âge : 'The medieval rule was to make a difference of twenty per cent from margin to margin', ${ }^{24}$ alors que 'the modern printer, in the teeth of the evidence given by his own eyes... prints the page in the middle of his paper'. ${ }^{25}$ 
Concrètement, la règle des vingt pour cent aboutit à des marges semblables à celles du dessin de la figure 3, repris de 'Printing'.

Figure 3 : Noter les proportions identiques aux bordures de la figure 1 .

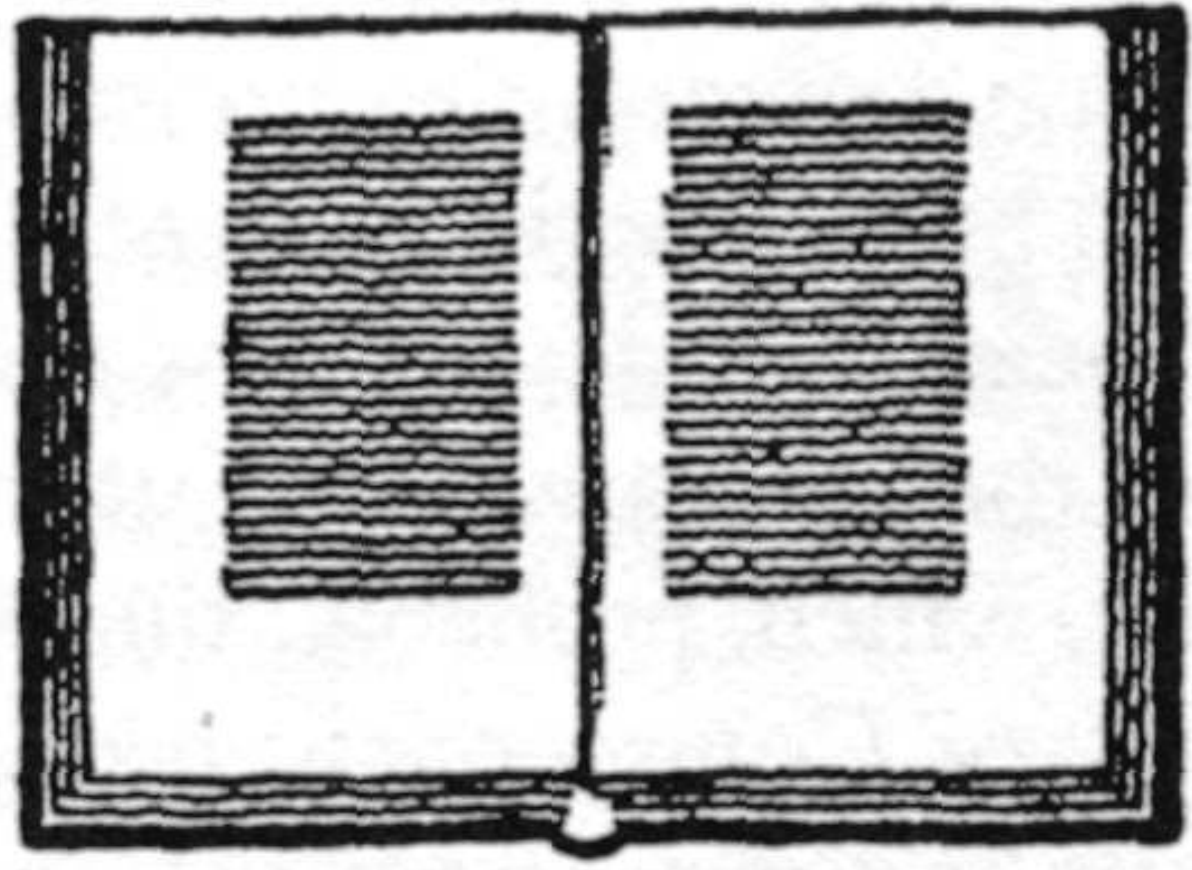


Figure 4 : Première page de The Works of Geoffrey Chaucer (Kelmscott Press, 1896).

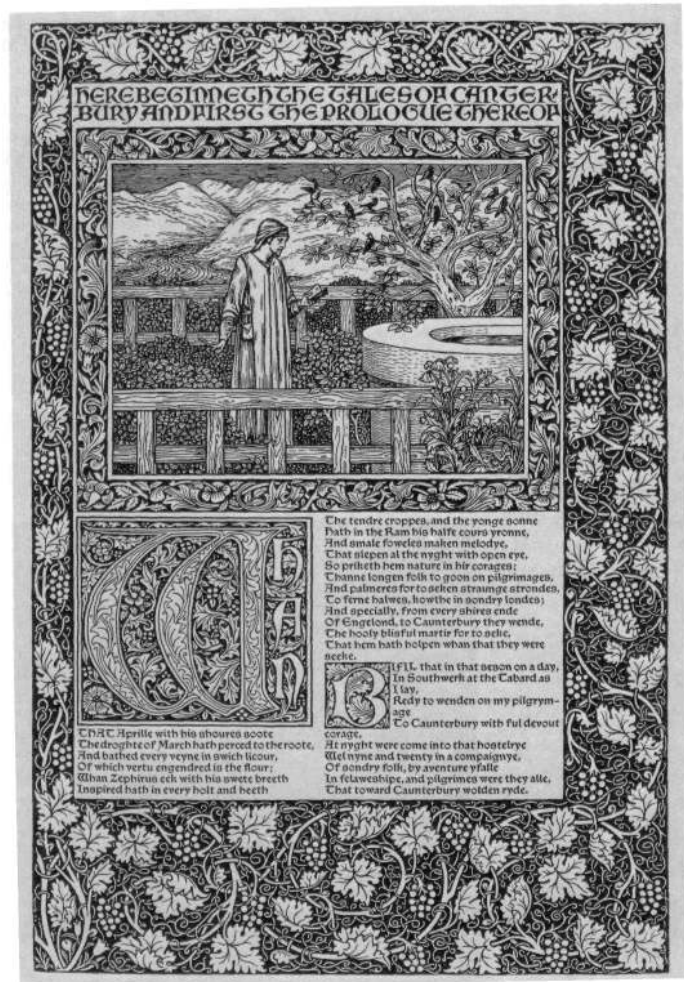

Le titre est en Troy Type, corps 18, le caractère gothique mis au point pour la Kelmscott Press, et le texte en Chaucer Type, corps 12, version réduite du précédent. Le dessin est de Burne-Jones, les bordures, les cartouches et la mise en page de William Morris. Pour ce qui est de la lisibilité, à la difficulté de l'anglais archaïque s'ajoute l'inaccoutumance du lecteur non spécialiste aux techniques graphiques médiévales.

Pour William Morris, bien sûr, 'these matters of spacing and position are of the greatest importance in the production of beautiful books... They will make a book printed in quite ordinary type at least decent and pleasant to the eye'. ${ }^{26}$

Mais là encore, ses critères pour décider de ce qui est «agréable à l'œil » sont, à l'évidence, fort éloignés des nôtres. Il est bien difficile de soutenir qu'il n'y a pas « perte de clarté » dans le manque d'espace entre les mots du titre de la première page du Chaucer. De même, ces pages nous apparaissent bien souvent surchargées quand il n'y a pas de passage en vers de moins d'une ligne ou de nouveaux paragraphes pour aérer l'ensemble (voir fig. 4). Cette 'solidity' nous semble aujourd'hui contraire à son objectif de clarté et de lisibilité. C'est bien sûr que les critères de lisibilité de William Morris sont l'aboutissement d'un processus mental différent du nôtre. Est «illisible " pour lui un livre qui heurte son sens de la beauté dérivé des édifices médiévaux. Le foisonnement de l'ornementation des pages du Chaucer ou de sa reliure en peau de truie travaillée au petit fer rappelle inévitablement les façades des cathédrales du Moyen Â ge, où aucune partie visible ou invisible ne reste sans décor. 
Figure 5 : Cartouche initial de la section 'An A.B.C. of Geoffrey Chaucer'.

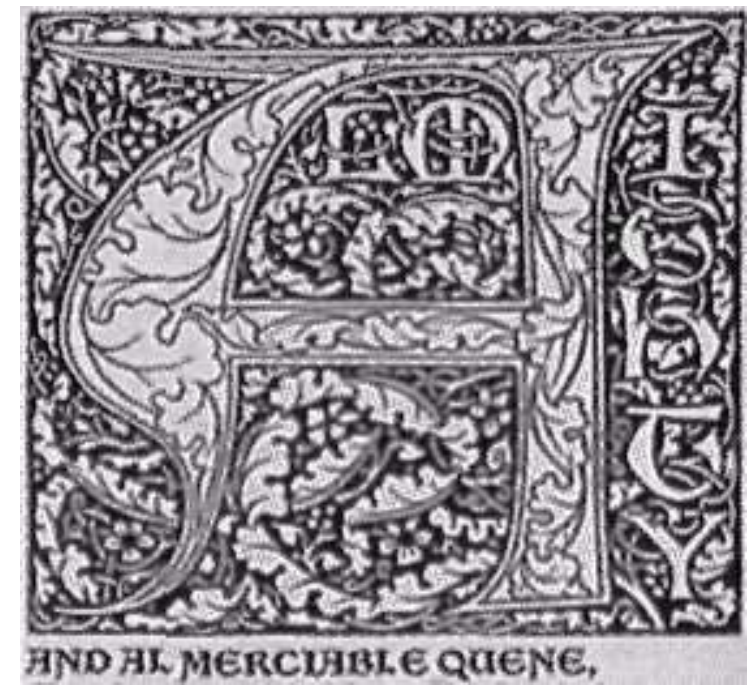

On peut donc avancer qu'en matière de graphisme les produits de la Kelmscott Press de William Morris constituent la traduction du Renouveau gothique dans le domaine du livre. Sans entrer dans des détails sur ce Renouveau gothique qui nous éloigneraient trop de notre sujet, on peut tout de même méditer le parallèle entre les critères retenus par William Morris pour ses livres et les critères de reconnaissance du style gothique donnés par Ruskin dans The Stones of Venice (1851-1853) au chapitre 'The Nature of Gothic' : 'It is not enough that it has the Form, if it have not also the power and life. It is not enough that it has the Power, if it have not the form'. ${ }^{27}$

\section{L'art calligraphique}

Mais, alors qu'aucun architecte d'aujourd'hui n'adhère plus à ce mouvement, dans l'art typographique, les conceptions de William Morris sont toujours vivantes, à tel point que l'on peut se procurer sans difficulté en ce début de XXI ${ }^{\mathrm{e}}$ siècle des polices pour imprimante d'ordinateur qui se veulent la réplique exacte des caractères qu'il a créés au XIX ${ }^{e}$ siècle $^{28}$.

17 On sait que les caractères d'imprimerie des pays à alphabet latin se rangent, pour simplifier, en deux grandes catégories : les gothiques et les romains. Les gothiques sont tout droit dérivés de la calligraphie des scribes médiévaux, et lorsque Gutenberg fabriquera ses premiers caractères mobiles, c'est d'eux qu'il s'inspirera tout naturellement ${ }^{29}$. Or William Morris était un très grand calligraphe lui-même - il avait très tôt fait œuvre de copiste et d'enlumineur pour certains de ses propres écrits, après avoir bien évidemment assimilé l'art de ses lointains prédécesseurs dans des manuscrits médiévaux, comme l'explique excellemment Fiona McCarthy :

Between 1870 and 1875 he worked on eighteen manuscript books and many trial fragments, a total of over 1,500 pages of laborious handwriting combined with a mass of decorative detail... With his revival of the skills of calligraphy and illumination Morris was, once again, entering new territory. These were techniques that had lain virtually fallow since the invention and development of printing in the fifteenth and sixteenth centuries... Morris's initiative turned this by then desiccated craft into an art form... It developed from his academic knowledge of the French and English mediaeval painted manuscripts in the Bodleian at Oxford and 
the British Museum, studied through the years with a hungry concentration... May Morris later claimed that nobody in London, except possibly the staff of the department, knew the British Museum Manuscripts collection in as much detail as her father did. ${ }^{30}$

En fait, il semble bien que ses toutes premières tentatives remontent aux années 1856-1857, à la suite de la conférence donnée par Ruskin sur 'The Distinction between Illumination and Painting' en novembre 1854, comme l'a établi Evelyn Phimister, qui retrouve dans le style de Morris les canons exposés par Ruskin: 'These early illuminated pages and their Gothic style imply not only an awareness of Ruskin's lectures but a sympathy with the ideas expressed there'. ${ }^{31}$ De plus, les années 1853-1854 sont celles où Morris a selon son biographe Mackail le plus intensément étudié les manuscrits médiévaux enluminés de la Bibliothèque bodléienne. ${ }^{32}$

Figure 6 : Feuille de vélin calligraphiée, enluminée et coloriée pour les Odes d'Horace.

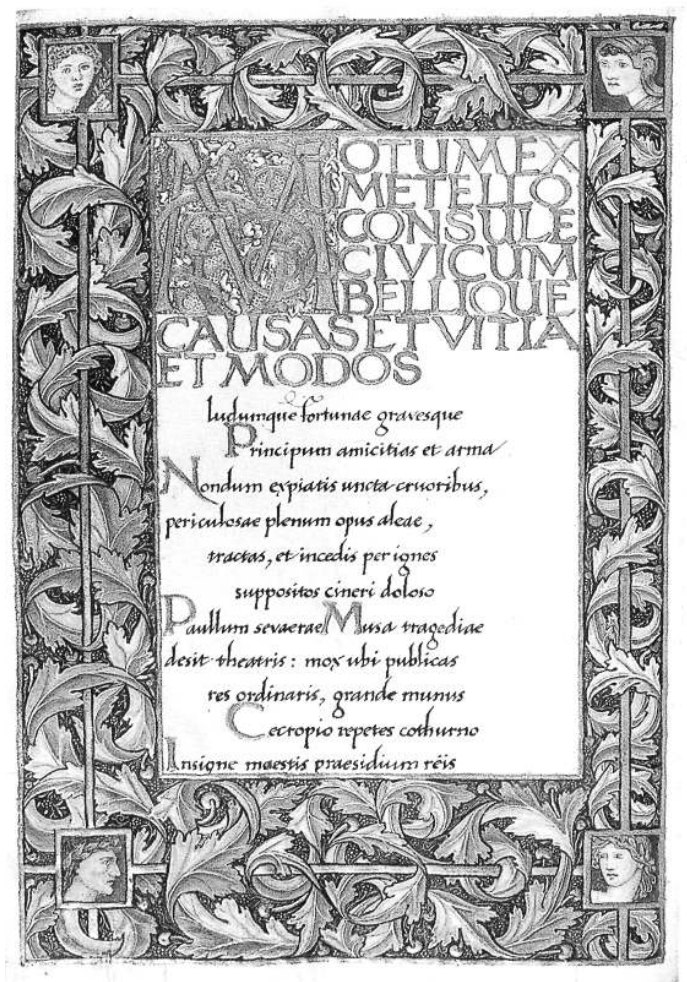

19 On ne s'étonnera donc pas de voir ces enluminures de la première manière de William Morris représenter en fait une imitation avouée de leurs aïeules gothiques. Comme tout grand artiste, il s'en éloignera peu à peu tout en en conservant l'essence. Si l'on ne craignait pas le mauvais jeu de mot, on pourrait dire que Morris en abandonnera la lettre tout en en perpétuant l'esprit. On peut en effet soutenir que le fil conducteur - la référence constante à la nature - reste invariable : seule varie la traduction graphique, Morris substituant de plus en plus ses propres inspirations végétales aux inspirations animales des ouvrages médiévaux comme thème dominant de l'ornementation. Si son Book of Verse illuminé de 1870 ou le décor de la reliure de Love is Enough (1873) montrent l'influence florale italianisante, avec la calligraphie du manuscrit illuminé des Odes of Horace de 1876 (premières esquisses en 1874 - voir figure 6), William Morris semble avoir trouvé la synthèse entre les traditions romaine et gothique. 

de l'esprit, une phrase de la conférence de Ruskin sur la responsabilité de l'enlumineur continuera sans nul doute d'imprégner le Morris de la maturité, a fortiori au cours des dernières années, celles de la Kelmscott Press : 'His object in introducing illuminations into books was not to lead the mind away from the text, but to enforce it'. ${ }^{33}$ Cette formule est à rapprocher de ce qu'écrira Morris lui-même près de quarante ans plus tard: 'The mediaeval craftsman had two sides to his artistic mind, the love of ornament, and the love of story. ${ }^{34}$

21 Il semble à cet égard que Paul Thompson fasse un contresens sur l'importance de William Morris en tant qu'enlumineur quand il écrit :

Contrary to what is probably the common impression, Morris's venture in illumination was not in itself original. The tradition, however debased, had never died out in formal documents such as addresses and charters. Its revival was a natural offshoot of the gothic revival and of the growth of monasticism in the nineteenth century. In addition, illuminating became a popular middle class Victorian hobby, more attractive to men than embroidery, but equally timeconsuming. ${ }^{35}$

En effet, c'est là ne pas voir que pour Morris, l'enluminure, pas plus d'ailleurs que n'importe quelle activité, n'a aucun sens en soi si elle fonctionne dans l'abstrait, en tant qu' « art d'agrément » au même titre que la broderie. Ce qui lui donne sa noblesse, et ce qui intéresse Morris, c'est bien sûr son intégration dans le grand projet artistique et artisanal que constitue la production d'un livre digne de ce nom. Peterson ne va à cet égard pas assez loin dans sa réponse implicite à Thompson quand il écrit :

In reality, Morris, with great independence of mind, charted his own course: the similarities between his manuscripts and the illuminated Biblical texts and uplifting proverbs done by middle-class Victorian ladies for display on the walls of their parlours are not very conspicuous. ${ }^{36}$

À partir du constat que l'introduction de l'imprimerie (mécanique) a introduit une rupture avec l'ornementation (faite main), William Morris va s'engager dans une nouvelle quête, la réunification du travail sur les caractères et du travail sur le décor du livre au sens large. Avant Gutenberg, cette unicité allait selon lui de soi. À la Renaissance (qu'il appelle 'New Birth' pour mieux s'en moquer par antiphrase), elle était déjà perdue :

The new German art spread like wildfire into every country of Europe; and in a few years written books had become mere toys for the immensely rich... By about 1530 all book illumination of any value was over, and thus disappeared an art which may be called peculiar to the Middle Ages, and which commonly shows mediaeval craftsmanship at its best, partly because of the excellence of the work itself, and partly because that work can only suffer from destruction and defacement, and cannot, like mediaeval buildings, be subjected to the crueller ravages of 'restoration' ${ }^{37}$

Son grand et noble dessein sera donc de réintroduire la conception globalisante des arts du livre, perdue à cause de la parcellisation capitaliste du travail. À partir de la Renaissance, la division des tâches entre l'ouvrier imprimeur et l'artiste ${ }^{38}$ illustrateur, tous deux stipendiés par l'entrepreneur/exploiteur, se reflète trop souvent dans la piètre qualité de l'ornementation, 'clearly the work of men employed to fill up a space, and having no interest in their work save livelihood'. ${ }^{39}$ Mais pour ce faire, il ne peut compter sur les éditeurs «commerciaux» de son temps, même ceux qui comme Charles T. Jacobi, 
directeur de la Chiswick Press, publient ses œuvres en tenant compte de ses avis esthétiques.

\section{L'art typographique}

Donc, sans être véritablement rebuté par les éditions commerciales de ses œuvres, il en est de plus en plus insatisfait. Il semble que ce soit avec les choix typographiques à faire pour la publication de The House of the Wolfings (Chiswick Press, 1889) qu'il intervienne pour la première fois en faisant procéder à différents essais en utilisant des polices différentes. Mais sa sélection est limitée par un obstacle insurmontable : l'éventail des polices disponibles sur le marché.

William Morris va par conséquent fonder sa Kelmscott Press, à la fois - comme c'était encore courant à l'époque - atelier d'imprimerie et maison d'édition, ce qui lui donne la maitrise complète de sa production, la distribution se faisant le plus souvent par souscription directe avant publication. William Morris se plonge dans la technique de la fabrication de livres "à l'ancienne » avec le même souci de recréation de la tradition que celui qu'il avait naguère manifesté pour les arts de l'ameublement au sein de Morris, Marshall, Faulkner \& $\mathrm{Co}^{40}$. O'Sullivan, s'appuyant sur le témoignage de May Morris (1934), décrit non sans humour les conséquences inattendues de ce culte de la perfection artisanale sur ses concitoyens incrédules :

Morris insisted on authenticity, and would use only natural materials and dyes, and traditional techniques. At the Great Exhibition of 1862, attempts were made to disqualify The Firm's contributions, on the grounds that they were medieval artefacts, retouched! ${ }^{41}$

Pour les productions de la Kelmscott Press, Morris va une fois plus se mettre en quête de l' «authenticité » selon les mêmes critères. À cet égard, la rencontre avec Emery Walker est décisive, comme Morris le déclarera lui-même : 'I may tell you candidly, I was not much of a typographer before Mr. Walker took me in hand'. ${ }^{42}$ Leurs chemins se croisent pour la première fois non pas dans une imprimerie, mais dans le Metropolitan Railway qui les ramène tous deux à Hammersmith au retour d'un meeting socialiste à Bethnal Green. Emery Walker (1851-1933), imprimeur passionné par son métier qui s'est mis à son compte avec un associé en 1886 à Hammersmith sous le nom de 'Walker \& Boutall, Automatic \& Photographic Engravers', est un socialiste convaincu à qui Morris va bientôt demander de devenir secrétaire de la Section de Hammersmith qu'il a fondée pour la Democratic Federation. Walker restera un familier de Kelmscott House jusqu'à la mort de Morris $^{43}$, leurs discussions passionnées sur le socialisme se mêlant à leurs discussions passionnées sur l'art du livre, que Walker possède si bien qu'il fait une conférence désormais considérée comme historique ${ }^{44}$ à la Arts \& Crafts Exhibition de 1888 sur le sujet, illustrée par de remarquables photographies projetées à la lanterne magique, qui impressionnera décisivement Morris si l'on en croit le compte-rendu qu'en fait May, qui y assistait avec son père :

One after another the old printers passed before us, one after another their splendid pages shone out in the dark room - such things as Boccaccio's De Claris Mulieribus by John Zainer of Ulm, with its woodcuts which my father thought could not be excelled for romantic and dramatic force, ${ }^{45}$ Schoeffer's Psalter of 1457 , a Jenson of 1470, a Sweynheim and Pannartz Livy (Rome 1469) - books which moved him to repeat more than once that 'the first printed books were the best ever done - the first and the last of fine printing. ${ }^{46}$ 

prise dès ce soir-là : il faut qu'il fonde ses propres presses, avec des polices inspirées des merveilles qu'il vient de voir en gros plan. 'Let's make a new fount of type', aurait-il dit à Walker en rentrant de la conférence, allant jusqu'à lui proposer presque aussitôt de s'associer à lui au sein de la Kelmscott Press ${ }^{47}$. On peut peut-être voir une autre indication de la communauté de pensée entre Emery Walker et William Morris dans le fait que Walker précède Morris en ce qui concerne l'analogie entre le livre et l'édifice, car les notes qui nous parvenues de cette conférence comprennent des comparaisons que l'on retrouvera dans 'Some Thoughts on the ornamented Manuscripts of the Middle Ages' : 'Type and paper, may be said to be to a printed book, what stones or bricks, and mortar, are to architecture'. ${ }^{48}$

Morris voulant éditer ou rééditer ses propres œuvres et celles qu'il admire chez les autres ${ }^{49}$, les ouvrages à publier ne manquent pas, et la première chose à faire, c'est de déterminer un ordre de priorité, avant de procéder aux choix techniques, au premier chef les polices d'impression. Le premier volume auquel il s'attelle est une nouvelle édition en anglais, sous le titre de The Golden Legend, d'une hagiographie imprimée à Strasbourg aux environs de 1472-1474, rédigée en latin par le dominicain Jacques de Voragine et intitulée Legenda aurea sanctorum ${ }^{50}$. La première édition de The Golden Legend est due à Caxton (1483), en caractères gothiques. Morris décide de ne pas imiter servilement le modèle : son volume ne sera pas imprimé sur deux colonnes, et il sera en caractères romains inspirés des meilleurs incunables de Nicolas Janson, un Français de l'école vénitienne $e^{51}$. Ce sera là l'origine de l'une des deux ${ }^{52}$ uniques polices créées et utilisées pour l'impression des ouvrages de la Kelmscott Press : le Golden Type. William Morris en élabore la forme grâce aux agrandissements photographiques que Walker lui fait spécialement, et transmet ensuite ses dessins à Edward Prince, le meilleur graveur de poinçons sur la place de Londres.

\section{William Morris, maître d'œuvre}

Nous abordons là une question qui a été trop souvent omise par les admirateurs de William Morris, à savoir la nature collective de la production de ses livres. Morris est avant tout un concepteur, qui laisse l'exécution concrète à des spécialistes. Ce qui fait de lui un éditeur hors du commun à la fin du XIX siècle, c'est que justement, il n'est satisfait que si ses collaborateurs, fournisseurs ou artisans sont les meilleurs qu'il ait pu trouver ${ }^{53}$, afin de pouvoir leur faire entièrement confiance non pas pour obéir sans réfléchir à ses instructions, mais pour savoir interpréter ses intentions dans leur domaine d'excellence. Emery Walker fait autorité en matière de sciences et techniques typographiques, et Morris lui laissera plus ou moins la responsabilité d'organiser le travail au jour le jour des ouvriers des presses à bras ${ }^{54}$, eux aussi recrutés pour leur «métier $»^{55}$. Edward Prince, comme l'a excellemment démontré John Dreyfus, photographies à l'appui ${ }^{56}$, interprétera plus qu'il ne copiera les dessins de Morris, affinant certains déliés, modifiant certaines courbes, rectifiant certains angles. Le point capital, qui loin de diminuer le mérite de William Morris le grandit encore, c'est qu'il s'incline de bonne grâce devant le savoir-faire de l'homme de métier qu'est Edward Prince. En d'autres termes, William Morris - chose rare en ce bas monde - pratique luimême ce qu'il prône :

Revue Française de Civilisation Britannique, XIII-1 | 2004 
I lay it down as a general principle in all the arts where one artist's design is carried out by another in a different material, that doing the work twice over is by all means to be avoided as the source of dead mechanical work. The 'sketch' should be as slight as possible, i.e., as much as possible should be left to the executant. ${ }^{57}$

William Morris ne rechignait pas autrefois à «mettre la main à la pâte ». Par exemple, il avait ourdi de sa main certaines de ses tapisseries de haute lisse, il avait expérimenté lui-même différentes recettes anciennes de teintures végétales pour mettre au point celles de Morris \& Co., mais il n'ira pas jusqu'à produire son encre, ni son papier, pas plus qu'à s'exercer à la reliure.

Pour l'encre, il ne trouve pas de fabricant à son goût en Grande-Bretagne, car aucun n'utilise plus l'huile de lin, à l'ancienne, comme base - la pétrochimie y ayant fait des " progrès » considérables, que Morris considère bien sûr comme autant de ravages. Il trouve une source allemande, Jaenecke Frères (Gebrüder Jänecke), à Hanovre - des socialistes comme lui, comme par hasard - qui acceptent ses exigences inspirées d'un autre âge :

Morris insisted that the oil should be freed from grease with stale bread and raw onions rather than chemicals, mixed with boiled turpentine, and matured for six months, after which the organic animal lampblack was ground into the mixture. ${ }^{58}$

Pour le papier, il a aussi des idées bien précises ${ }^{59}$, héritées d'avant la mécanisation. Il veut du pur chiffon de lin - et non de coton - pour la pâte. Il a horreur du papier vergé moderne, où le filigrane en forme d'échelles d'une régularité parfaite est produit artificiellement avec trop de facilité par les machines. Seules les feuilles produites à l'unité, «à la forme », selon la meilleure tradition, recueillent son approbation. Il va finir par trouver un fabricant britannique qui le satisfasse, Joseph Batchelor, installé à Chart, dans le Kent, à qui il apporte comme modèle de ce qu'il a en tête un échantillon de papier fabriqué à Bologne en $1473^{60}$. L'anecdote veut qu'il se soit essayé à la fabrication d'une feuille au cours de sa visite chez Batchelor, avec un plein succès lors de sa deuxième tentative - mais, nous l'avons vu, il en restera là, laissant le soin aux artisans papetiers de lui fournir les quantités considérables que nécessite la Kelmscott Press ${ }^{61}$.

Une autre anecdote veut qu'il ait été sur le point de demander au pape d'intercéder en sa faveur auprès des presses du Vatican pour qu'elles lui laissent une plus grande partie de la production de vélin ${ }^{62}$ de son fabricant italien, auprès duquel il se fournissait de longue date. En effet, les exemplaires de luxe sur vélin de The Golden Legend, avec leurs 1300 pages, allaient épuiser les réserves de Morris, et on pouvait penser que le pape serait prêt à faire un effort pour encourager la réimpression d'un pieux ouvrage de dévotion aux saints qui n'avait eu aucune édition anglaise depuis $1527^{63}$. Là encore, William Morris trouve finalement aussi bien auprès d'un fournisseur britannique, Henry Baud, de Brentford, dans le Middlesex. Comme pour le filigrane artificiel du papier, Morris récuse le blanc du vélin obtenu par cérusage. Le vélin servant également parfois pour la reliure des exemplaires tirés sur papier, Morris fait d'ailleurs relier ceux qui lui sont destinés avec les vélins que les amateurs au discernement moins prononcé que le sien rejetteraient : il prise les peaux qui conservent la trace brune des poils de l'animal - marque certaine du caractère naturel du traitement qu'elles ont connu. Vantant leurs qualités tactiles, Fiona McCarthy écrit fort justement : 'To understand their quality you need to hold and stroke these Kelmscott books in their limp vellum covers just as Morris used to clasp his mediaeval incunabula'. ${ }^{64}$ 
La reliure proprement dite est confiée à un ami, T.J. Cobden-Sanderson (1840-1922), ancien avocat devenu relieur passé l'âge de quarante ans, apparemment à la suite d'une conversation en 1883 avec Jane, la femme de William Morris. La première reliure qu'il fera pour lui, en 1884, sera celle de son exemplaire du Capital de Karl Marx, qui tombait en morceaux à force d'être consulté. Mais alors que Philip Webb la trouve très belle ${ }^{65}$, il semble que Morris reste indifférent à l'art de Cobden-Sanderson, qui écrit dans ses Carnets : "[Morris] thought my work too costly; bookbinding should be "rough"; did not want to multiply the minor arts (!); went so far as to suggest that some machinery should be invented to bind books'. ${ }^{66}$ Cette suggestion, qui constitue bien évidemment le comble de l'abomination aussi bien pour Cobden-Sanderson ${ }^{67}$ que pour Morris, montre clairement l'évolution de ce dernier : en 1884, sept ans donc avant la fondation de la Kelmscott Press, sa définition personnelle des arts du livre n'englobe pas encore la reliure ${ }^{68}$. Ce serait par conséquent un contresens que de lui prêter dès sa jeunesse l'omniscience en matière d' « arts mineurs " ${ }^{69}$ : il y a au contraire dans la fondation de la Kelmscott Press comme l'aboutissement d'un processus de maturation, d'un élargissement des perspectives de Morris dans le domaine des « arts mineurs » liés au livre, d'une prise de conscience finalement tardive qu'un «beau livre $»^{70}$ est un tout ${ }^{71}$, menant à cette quête du Gesamtkunstwerk que nous voyons chez lui.

On oublie trop souvent également que si les lettres enluminées et les bordures florales sont de William Morris, la plupart des dessins « figuratifs " ne sont pas de lui, mais de Burne-Jones, à qui il laisse carte blanche, par exemple pour The Works of Geoffrey Chaucer $^{72}$. Enfin, il faut se souvenir que Morris, malgré son amour incontestable des textes anciens qu'ils publie, laisse encore une fois à d'autres qu'il juge plus qualifiés que lui le soin de mettre au point la version qui semble la meilleure à partir des variantes qui nous sont parvenues. Pour une quinzaine de volumes sortis de la Kelmscott Press, dont The Golden Legend et le Chaucer, ce sera F.S. Ellis, libraire-éditeur retraité et passionné par l'exactitude des textes, qu'il s'agisse d'établir l'édition originale ou de relire les épreuves pour ne laisser aucune erreur. Pour d'autres, comme The Recuyell of the Historyes of Troye, ce sera son gendre, H.H. Sparling.

L'erreur à ne pas commettre est celle que fait Bradley quand il écrit : 'He had regarded himself first and foremost as a designer - he had described himself thus on his membership card for the Democratic Federation - and it was in this sphere that his influence was most perceptible and immediate. ${ }^{73}$ En effet, William Morris ne se contente pas de concevoir la « maquette » des livres de la Kelmscott Press, et c'est beaucoup trop réducteur de voir en lui un simple 'designer' - même si c'est ainsi qu'il choisissait de se décrire lui-même. Certes, nous venons de le voir, il n'est pas non plus le réalisateur unique des ouvrages qui en sortent. Mais au bout du compte, il apparaît clairement que dans la réalisation du «beau livre » de Morris la comparaison avec l'architecte peut être poussée jusqu'à sa limite extrême. Quand on parle de la Joconde de Léonard de Vinci, on entend bien exprimer que c'est lui qui a peint le tableau de ses mains : le concepteur est à la fois réalisateur/exécutant. Le compositeur de musique symphonique est déjà dans une situation différente, puisqu'il lui est impossible d'exécuter ses créations lui-même, même s'il est aussi chef d'orchestre. L'architecte est dans le même cas : quand on fait allusion à l'Opéra de Garnier, il y a ambiguïté, car si Garnier en est bien le concepteur, s'il a bien dirigé les travaux de construction en qualité d'architecte, il ne l'a pas édifié de sa main. De même, William Morris, indubitable créateur des livres de la Kelmscott Press, en est l'architecte, le coordonnateur, le maître d'œuvre - mais on ne peut pas 
parler du Chaucer de William Morris au même titre qu'on parle de la Joconde de Léonard de Vinci. Et il ne s'agit pas d'une question d'auteur du texte. Si l'on parle du News from Nowhere de la Kelmscott Press, il faut distinguer entre l'auteur du texte (Morris), le concepteur du volume (Morris) et les réalisateurs du livre proprement dit (tout un éventail de spécialistes, où Morris est minoritaire). Tout cela n'enlève rien bien entendu au mérite créatif de William Morris, pas plus qu'on ne reproche à Garnier de ne pas avoir construit l'Opéra lui-même, mais ces précisions de vocabulaire montrent bien l'analogie avec l'architecte maître d'œuvre plus qu'avec le peintre qui réalise luimême ses tableaux.

\section{Conclusion : les contradictions de William Morris}

Parallèlement aux reproches qui lui ont été faits sur le prix de ses livres, les détracteurs de William Morris n'ont pas manqué de remarquer qu'il enfreignait ses propres règles quand cela lui plaisait ${ }^{74}$. L'exemple « technique » le plus souvent cité, quand on parle de la Kelmscott Press, c'est celui du recours à la photographie pour la création de ses caractères $^{75}$. De plus, il «fait marcher le commerce capitaliste » en commandant son papier ou son vélin à des entreprises pleinement intégrées dans l'économie de marché, et la Kelmscott Press elle-même repose bien sûr sur le salariat, "l'exploitation de l'homme par l'homme ", même s'il semble que ses ouvriers imprimeurs aient été mieux payés qu'ailleurs ${ }^{76}$. Pearson explique cependant très bien pourquoi on peut douter de la pertinence de ces critiques :

Morris, like Marx, did not believe in the need for personal 'examples'. He fought for a change in society which would deprive him of the capital which enabled him to pursue his artistic ambitions, but such a change would create the circumstances which he longed for to make his artistic values a natural part of society. In the meantime, the Kelmscott Press and Morris \& Co. were not 'compromises' but the only available form for his social artistic ambition. ${ }^{77}$

D'autres veulent juger la Kelmscott Press en creux, n'y voyant à la limite qu'un pis-aller après l'échec de Morris comme militant politique ${ }^{78}$. Reste évidemment la controverse inépuisable sur la nature "victorienne " ou "anti-victorienne» de la démarche de William Morris, que Fiona McCarthy quant à elle évacue par une habile pirouette qui n'éclaire guère son lecteur: 'These books are Victorian and yet anti-Victorian'. ${ }^{79}$ Peterson fait pour sa part une remarque de bon sens, qui rappelle que comme tout un chacun Morris est prisonnier de son temps: 'The Troy type is in reality as characteristic a product of the Gothic Revival in Victorian England as Pugin and Barry's Palace of Westminster or Butterfield's All Saints Church'. ${ }^{80}$ Tout ce que nous savons de lui donne à penser qu'il était conscient de ce statut de prisonnier - reste à s'accorder sur les moyens qu'il a voulu se donner pour sa délivrance, et même ses contempteurs les plus sévères admettront que le travail (au sens très large qu'il donnait à ce terme) venait au premier rang. Constatant une évolution plutôt qu'une contradiction, ses admirateurs verront dans le «beau livre » tel qu'il sort des célèbres presses de Hammersmith l'aboutissement de sa conception du travail qu'il évoquait dès l'âge de vingt-deux ans: 'My work is the embodiment of dreams in one form or another' ${ }^{81}$

CEuvre d'art totale? Peut-être pas; mais réalisation tangible d'un rêve artistique intangible - sans aucun doute. La modeste devise héritée du Moyen Âge qui figure sur les vitrages et les carrelages muraux de Red House (1859), "Si je puis", a 
indiscutablement trouvé sa concrétisation dans les arts du livre tels que William Morris les a pratiqués, au-delà de toutes les contradictions que l'on se plaira à souligner.

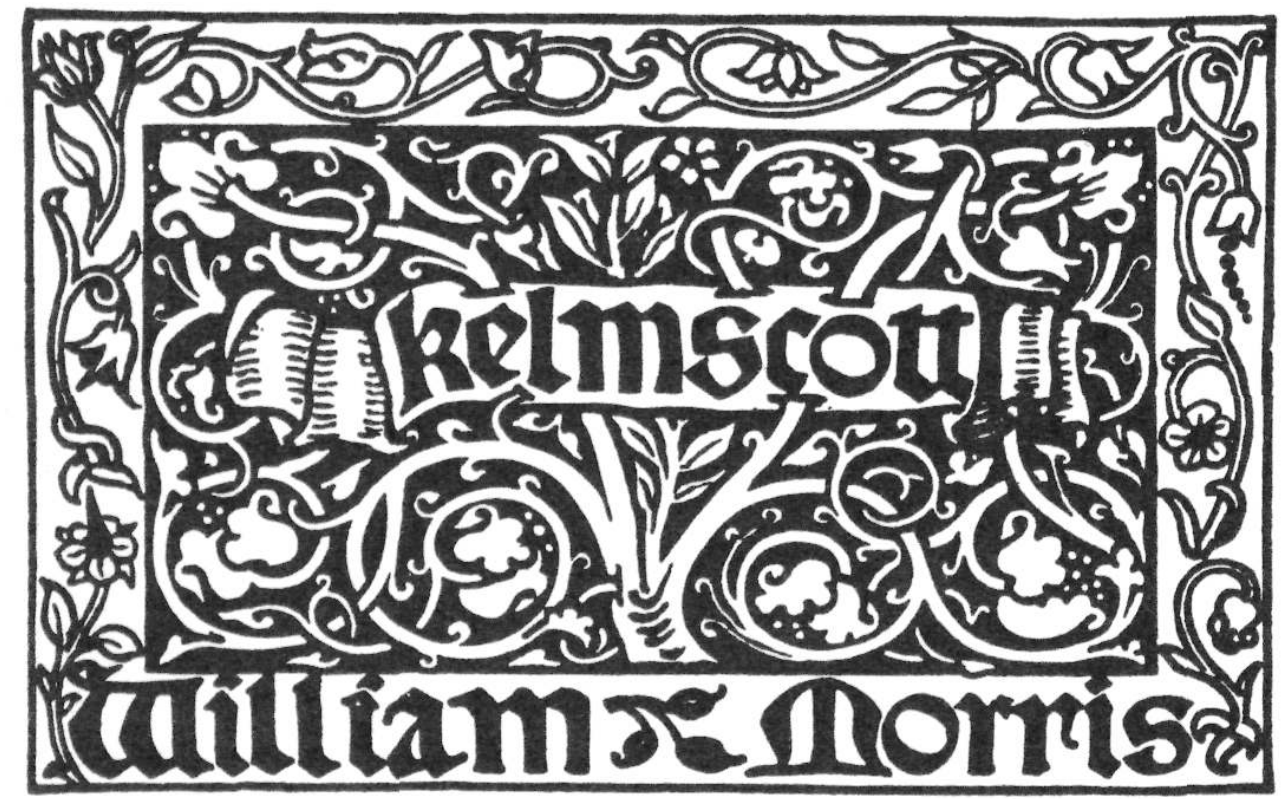

\section{NOTES}

1. Pour l'opinion - très négative - de Morris (contrairement à ses amis Burne-Jones et Bernard Shaw) sur Wagner, notamment son traitement de Sigurd/Siegfried dans la tétralogie, voir Philip HENDERSON, William Morris : His Life, Work and Friends, London: Thames \& Hudson, 1967, pp. 168-170.

2. Le présent article poursuit - en y ajoutant certaines illustrations - une réflexion entamée naguère dans le deuxième numéro "papier » de CERCLES, publié par ce qui s'appelait alors le Centre d'études en littérature, linguistique et civilisation de langue anglaise (CELLCLA). Antoine CAPET, «William Morris et le 'Beau Livre' : Quelques réflexions à l'occasion du centenaire de la Kelmscott Press », CERCLES n², hiver 1991, pp. 41-57.

3. Elle cite là Norman KELVIN (ed.), The Collected Letters of William Morris. 4 vol. Princeton: Princeton University Press, 1984-1996, vol. II., p. 52.

4. Isolde Karen HERBERT, "The "Sympathetic Translation" of Patterns : William Morris as Singer, Scribe, and Printer', Journal of the William Morris Society, vol. XIII, n ${ }^{\circ}$, Spring 2000, pp. 21-29 (p. 21).

5. William S. PETERSON fait à bon droit une remarque qui contredit ces propos : 'But in Morris's News from Nowhere (1891), a vision of a Socialist utopia in the twenty-first century, the British Museum has been largely abandoned, and very few Englishmen display an interest in books.' The Ideal Book : Essays and Lectures on the Arts of the Book by William Morris, édition et introduction de William S. PETERSON, Berkeley \& Los Angeles: University of California Press, 1982, p. 125.

6. 'The Poet as Printer'. Entretien avec un journaliste non identifié publié dans la Pall Mall Gazette, 12 November 1891, pp. 1-2. Reproduit dans William S. PETERSON, The Ideal Book, pp. 89-95 (p. 92). 
7. May MORRIS, The Introductions to the Collected Works of William Morris, Edited by Joseph R. Dunlap, 2 vol., New York: Oriole Editions, 1973, vol. 1, p. 39.

8. 'The Poet as Printer'. William S. PETERSON, The Ideal Book, p. 94.

9. William S. PETERSON. The Kelmscott Press : A History of William Morris's typographical Adventure, Oxford: Clarendon Press, 1991, p. 79.

10. Fiona McCARTHY, William Morris : A Life for our Time, London: Faber \& Faber, 1994, p. 608.

11. Nous avons là-dessus le témoignage de Cockerell : 'It was at this time that William Morris began to collect the mediceval books of which he formed so fine a library in the next six years. He had made a small collection of such books years before, but had parted with most of them, to his great regret. He now bought with the definite purpose of studying the type and methods of the early printers.' Sydney COCKERELL, 'A short History and Description of the Kelmscott Press' (Addendum à la réédition posthume de $A$ Note by William Morris on his Aims in founding the Kelmscott Press, London: Kelmscott Press, 1898). Reproduit dans William S. PETERSON, The Ideal Book, pp. 79-88 (p. 83). Peterson a également mis le texte de Cockerell sur son site :

<http://www.english.umd.edu/englfac/WPeterson/MFP/documents/18.html>

12. Rappelons qu'on entend par incunable un livre publié entre 1450 (introduction de l'imprimerie à caractères mobiles par Gutenberg) et 1500 .

13. Conférence prononcée devant la Bibliographical Society le 19 juin 1893 et publiée dans les Transactions of the Bibliographical Society, $\mathrm{n}^{\circ} 1,1893$, pp. 179-186. Reproduite dans William S. PETERSON, The Ideal Book, pp. 67-73.

14. Conférence 'The Ideal Book', in William S. PETERSON, The Ideal Book, p. 67.

15. Susan Otis THOMPSON, American Book Design and William Morris, New York \& London: Bowker, 1977, pp. 223-229. (Second expanded edition. Foreword by Jean-François Vilain. London: British Library \& New Castle (Delaware): Oak Knoll Press, 1996).

16. 'Some Thoughts on the Ornamented Manuscripts of the Middle Ages'. (Manuscrit datant probablement de 1892. Publication posthume à partir du texte déposé à la Huntington Library, New York: Press of the Woolly Whale, 1934.) Reproduit dans The Ideal Book, op. cit., pp. 2-6 (p. 2). (Reproduit également en partie dans Christine POULSON (ed.), William Morris on Art \& Design. Sheffield: Sheffield Academic Press, 1996, p. 142.)

17. Noter l'« hénaurme » coquille de Robinson, chez qui le 'fine building' de la citation devient 'a fine binding'. Duncan ROBINSON, William Morris, Edward Burne-]ones and the Kelmscott Chaucer, London: Gordon Fraser, 1982, p. 36.

18. Conférence 'The Ideal Book', in William S. PETERSON, The Ideal Book, p. 67 ; p. 73.

19. William MORRIS, 'A Note by William Morris on his Aims in founding the Kelmscott Press', Modern Art, $\mathrm{n}^{\circ}$ 4, Winter 1896, pp. 36-39. Reproduite dans William S. PETERSON, The Ideal Book, pp. 75-78 (p. 75). Peterson a également mis le texte de Morris sur son site :

<http://www.english.umd.edu/englfac/WPeterson/MFP/documents/9.html>

20. William MORRIS, 'Printing'. In Arts \& Crafts Essays - by members of the Arts and Crafts Exhibition Society; with a preface by William Morris, London: Rivington, Percival, 1893. Reproduit dans William S. PETERSON, The Ideal Book, pp. 59-66 (p. 62).

21. L'objet du présent article n'est pas de traiter de la pensée générale de William Morris. Rappelons cependant ces fortes paroles de 1877 : 'Nothing can be a work of art which is not useful'. Conférence The Lesser Arts donnée à la Trades' Guild of Learning, 4 décembre 1877. Reproduite dans News from Nowhere and Other Writings. London: Penguin, (Revised) 1998, pp. 231-254 (p. 251). Notons au passage qu'il n'y parle pas des arts et métiers du livre, confirmant ainsi que son intérêt latent ne s'est pas encore concrétisé en 1877.

22. William S. PETERSON, The Ideal Book, p. 69.

23. William MORRIS, 'Printing', p. 62.

24. 'A Note by William Morris on his Aims in founding the Kelmscott Press', op. cit., p. 78.

25. William MORRIS, 'Printing', p. 64. 
26. 'A Note by William Morris on his Aims in founding the Kelmscott Press', op. cit., p. 78.

27. John RUSKIN (1819-1900), 'The Nature of Gothic' (1851-1853). In Selections from the Writings of John Ruskin, London: Melrose, 1907, p. 236.

28. Notons également avec Coote l'influence à long terme de ses expériences calligraphiques : 'These experiments were also to be highly influential since they were to impress those who later guided Edward Johnston [1872-1944], the great modern pioneer of revised calligraphy'. Stephen Coote, William Morris : His Life and Work, London: Garamond, 1990 (Stroud: Sutton, 1996, p. 88).

29. «Les premiers livres imprimés ressemblaient tellement à des manuscrits qu'on les crut d'abord sortis de la main des copistes ". Émile LECLERC, Nouveau manuel de typographie. Encyclopédie Roret, Paris : S.F.E.L.T., 1939, p. 19.

30. Fiona McCARTHY, William Morris : A Life for our Time, p. 265.

31. Evelyn J. PHIMISTER, 'John Ruskin, William Morris, and the illuminated Manuscript', Journal of the William Morris Society, vol. XIV, $\mathrm{n}^{\circ} 1$, Autumn 2000, pp. 30-36 (p. 30 ; p. 32).

32. 'One of these, a splendid Apocalypse of the thirteenth century, became his ideal book. Forty years later he went to Oxford to spend a day in studying it, and looked over it with greater knowledge but unimpaired satisfaction'. J.W. MACKAIL, The Life of William Morris, 2 vol. London: Longmans, 1899 (New York: Haskell House, 1970, vol.1, p. 42).

33. Cité par Evelyn J. PHIMISTER, 'John Ruskin, William Morris, and the illuminated Manuscript', p. 32 .

34. 'Some Thoughts on the ornamented Manuscripts of the Middle Ages'. Reproduit dans William S. PETERSON, The Ideal Book, p. 2.

35. Paul THOMPSON, The Work of William Morris, London: Heinemann, 1967, p. 134.

36. William S. PETERSON, The Kelmscott Press, p. 59.

37. 'Some Thoughts on the Illuminated Books of the Middle Ages'. Magazine of Art, $\mathrm{n}^{\circ} 17$ (January 1894), pp. 83-88. Reproduit dans William S. PETERSON, The Ideal Book, pp. 7-14 (pp. 13-14).

38. Dans sa conférence prononcée à plusieurs reprises entre 1884 et 1886, The Relations of Art to Labour, William Morris dira : 'The craftsmen were now divided into artists who were not workmen, and workmen who were not artists'. William MORRIS, The Relations of Art to Labour, London: Cooperative Wholesale Society, 1890 (Edited with an Introduction by Alan BACON and an Appendix by Lionel C. YOUNG, London: William Morris Society, 2004, p. 33). Voir également son article paru dans Commonweal (Vol. 3, $\mathrm{n}^{\circ}$ 87, 10 September 1887), 'Artist and Artisan as an Artist sees it', où il écrit notamment : 'Before the rise of capitalism in the sixteenth century, the artisan did not differ in kind from the artist; all craftsmen who made anything were artists of some kind, they only differed in degree'. (Reproduit dans William MORRIS, Political Writings : Contributions to Justice and Commonweal, 1883-1890, p. 276.)

39. 'Some Thoughts on the illuminated books of the Middle Ages', p. 14.

40. 'The Firm', comme il l'appelait, fondée en 1861 et rebaptisée simplement Morris \& Co. en 1874.

41. Paddy O'SULLIVAN, 'Who was William Morris?', in Stephen COLEMAN \& Paddy O'SULLIVAN (eds.), William Morris \& News from Nowhere : A Vision for our Time. Bideford: Green Books, 1990, p. 19. 42. 'The Kelmscott Press : An illustrated Interview with Mr. William Morris'. Entretien avec un journaliste (identifié seulement par les initiales I.H.I.) publié dans Bookselling (Christmas 1895), pp. 2-14. Reproduit dans William S. PETERSON, The Ideal Book, pp. 106-117 (p. 110).

43. 'Emery Walker, of whom Morris said that he regarded that day as lost on which he did not see him'. Philip HENDERSON, William Morris : His Life, Work and Friends, p. 318.

44. Conférence intitulée Letterpress Printing and Illustration, 15 novembre 1888. Peterson écrit d'elle : 'Many historians have argued that the modern revival of fine printing can be dated precisely from Walker's lecture'. William S. PETERSON, The Kelmscott Press, p. 74.

45. 'The Ulm printer, John Zainer, in especial shone in the production of borders. His De claris mulieribus excels all the other books of the school in this matter'. '...my own choice would be the De claris mulieribus, 
partly perhaps because it is a very old friend of mine, and perhaps the first book that gave me a clear insight into the essential qualities of the mediaeval design of the period'. William MORRIS, 'On the artistic Qualities of the Woodcut Books of Ulm and Augsburg in the fifteenth Century'. Bibliographica, $\mathrm{n}^{\circ} 1$, 1895, pp.437-455. Reproduit dans William S. PETERSON, The Ideal Book, pp. 45-58 (p. 48, p. 52). Une des ironies de l'Histoire, c'est que c'est William Morris ('virtually unknown in Germany during his lifetime') qui réintroduira indirectement cet art perdu en Allemagne. Cf. Hans ECKERT, 'The Impact of Morris and the Kelmscott Press in Germany', Journal of the William Morris Society, vol. XI, n², Spring 1995, pp. 20-24 (p. 20).

46. Citée par Fiona McCARTHY, William Morris : A Life for our Time, op. cit., p. 612.

47. Ibid., p. 612.

48. 'Appendix B : Lecture by Emery Walker'. In William S. PETERSON, The Kelmscott Press, pp. 324-331 (p. 325).

49. 'I thought I should like to see my own writings in the handsomest type, but apart from that I wished to print masterpieces in literature, and particularly to give a turn to early English classics like Caxton's'. ، "Master Printer Morris" : A Visit to the Kelmscott Press'. Entretien avec un journaliste non identifié publié dans la Daily Chronicle (22 February 1893), p.3. Reproduit dans William S. PETERSON, The Ideal Book, pp. 95-98 (p. 97). Charité bien ordonnée commençant par soi-même, la Kelmscott Press publiera en tout vingt-trois livres de William Morris, sur un total de cinquantetrois recensés par Sparling.

50. Euvre qui connut en son temps un succès considérable si l'on en croit Michel BRAUDEAU : «La Légende dorée - une collection de vies des saints, rédigée en latin dans la seconde moitié du XIII ${ }^{\mathrm{e}}$ siècle, qui fut, après la Bible, le plus grand best-seller du Moyen Âge ». "L'usage des saints », Le Monde, 25-26 juillet 2004, p. 8.

51. Nicolas Janson (1420?-1481), graveur envoyé auprès de Gutenberg à Mayence par Charles VII pour y étudier la technique nouvelle qu'était l'imprimerie, finira par se fixer à Venise en 1470. Il est connu en Grande-Bretagne (et donc de William Morris) sous le nom de Nicholas Jenson.

52. C'est à tort qu'on parle parfois de trois polices. En effet le Troy Type et le Chaucer Type ne sont que deux versions de corps différent du même caractère gothique. Dans les faits, la Kelmscott Press imprimait tout soit en romain (Golden Type, corps 14, alors appelé 'English'), soit en gothique (Troy Type, corps 18 ['Great Primer'], utilisé pour la première fois pour The Recuyell of the Historyes of Troye; ou Chaucer Type, corps 12 ['Pica'], réduction du précédent pour s'adapter sans disproportion disgracieuse aux doubles colonnes de The Works of Geoffrey Chaucer). À cause de sa passion indéfectible pour le gothique, William Morris préférait le Troy Type et le Chaucer Type au Golden Type.

53. 'No expense was spared in getting everything connected with it [the Kelmscott Press] as near his ideal as could be produced'. J.W. MACKAIL, The Life of William Morris, vol. 2, p. 260).

54. À noter que l'une des quatre presses, achetées d'occasion, a survécu et est exposée au siège de la William Morris Society, à Kelmscott House, Hammersmith.

55. Notamment William Henry Bowden, maître-imprimeur à la retraite, qui avait imprimé News from Nowhere pour Reeves \& Turner. Bowden a plus tard confié certains de ses souvenirs de Morris et de la Kelmscott Press à Sparling, qui les a publiés dans Henry Halliday SPARLING, The Kelmscott Press and William Morris, Master-Craftsman. Edited by Robert Steele. With 'An annotated list of all the books printed at the Kelmscott Press'. London: Macmillan \& Co., 1924.

56. John DREYFUS, Morris and the printed Book: A Reconsideration of his Views on Type and Book Design in the Light of later Computer-aided Techniques. The Sixth Annual Kelmscott Lecture of the William Morris Society, given at the Art Workers Guild on Wednesday $8^{\text {th }}$ October 1986. London: William Morris Society, 1989, figs. 3-6, pp. 16-20. Les figures reproduisent de façon synoptique et très agrandie les dessins de Morris et les contours des poinçons de Prince, ce qui met les notables différences en évidence. 
57. The Woodcuts of Gothic Books, conférence prononcée devant la Society of Arts le 26 janvier 1892 et publiée dans le Journal of the Society of Arts, n40, 12 February 1892, pp. 247-260. Reproduite dans William S. PETERSON, The Ideal Book, pp. 25-44 (p. 39).

58. Paul THOMPSON, The Work of William Morris, p. 142.

59. 'Our ideal book must, I think, be printed on hand-made paper as good as it can be made'. Conférence 'The Ideal Book', op. cit., p. 72.

60. 'A Note by William Morris on his Aims in founding the Kelmscott Press', op. cit., p. 75.

61. Rien que l'impression de The Golden Legend a nécessité une tonne et demie de papier : on peut imaginer le nombre d'heures de travail que la fabrication représente à la main, feuille par feuille.

62. On sait que le parchemin est fait avec des peaux d'agneau ou de chevreau, le vélin avec des peaux de veau, si possible mort-né.

63. Finalement, il n'y aura pas d'exemplaires imprimés sur vélin. Mais ce n'est que partie remise : les autres grands titres publiés par la Kelmscott Press auront tous un tirage spécial sur vélin, qui en fait monter considérablement le prix. À titre d'exemple, The Recuyell of the Historyes of Troye (grand in $-4^{\circ}, 1892$ ) coûte 9 guinées en version papier, 80 livres en vélin. News from Nowhere (in- $8^{\circ}$, 1892) passe de 2 à 10 guinées, comme Beowulf (1895). Le record est bien entendu détenu par The Works of Geoffrey Chaucer (in-folio, 1896) qui de 20 livres sur papier monte à 120 guinées en version vélin reliée en peau de truie. Les mauvaises langues ne manqueront pas de faire la comparaison avec les salaires ouvriers (il faut se souvenir que Seebohm Rowntree fixe à un peu plus d'une livre par semaine le revenu nécessaire à un ménage avec trois enfants en 1899 : une famille de cinq personnes pourrait alors vivre quelque deux ans avec le prix du Chaucer de luxe). Pour les tirages et les tarifs, voir 'Appendix A : Checklist of the Kelmscott Press Books', William S. PETERSON, The Kelmscott Press, pp. 315-323.

64. Fiona MCCARTHY, William Morris : A Life for our Time, 1994, p. 620. La bibliothèque de Kelmscott House en possède quelques-uns, que l'on peut manipuler.

65. Ibid., p. 592. Rappelons au passage que Webb (1831-1915) est l'architecte de Red House : une fois plus dans l'univers morrissien l'esthétique du livre recoupe l'esthétique de l'architecture.

66. T.J. COBDEN-SANDERSON, The Journals of Thomas James Cobden-Sanderson, 1879-1922, London: Richard Cobden-Sanderson, 1926, pp. 211-212, cité par Paul THOMPSON, The Work of William Morris, p. 137.

67. Cobden-Sanderson partage en effet pleinement les idées qui inspirent William Morris, à tel point que selon Mackail, c'est lui l'auteur d'une expression promise à un grand avenir : 'The name of the Arts and Crafts was the invention, at a somewhat later stage, of Mr. Cobden-Sanderson'. J.W. MACKAIL, The Life of William Morris, vol. 2, p. 210.

68. Pourtant, William Morris a indirectement contribué à créer l'école anglaise de la reliure artisanale. Cobden-Sanderson crée avec sa Doves Bindery (1893) un mouvement qui est toujours vivant aujourd'hui. En outre, Douglas Cockerell (1870-1945), bras-droit de Cobden-Sanderson et frère du secrétaire de la Kelmscott Press, Sydney Cockerell (1867-1962), écrit dès 1901 un manuel sur la reliure traditionnelle, c'est-à-dire héritée au bout du compte du Moyen Âge, dont s'inspirent encore actuellement ceux qui veulent respecter les techniques du livre remises à l'honneur par la Kelmscott Press : Douglas COCKERELL, Bookbinding, and the Care of Books : A TextBook for Book-Binders and Librarians. The Artistic Crafts Series of Technical Handbooks, edited by W.R. Lethaby. London: John Hogg, 1901 (nombreuses rééditions postérieures). La tradition se perpétue de nos jours dans la revue professionnelle de très haute tenue Designer Bookbinders.

69. C'est l'erreur que fait semble-t-il Lesley A. Baker en écrivant : 'Morris always made his aims very clear'. Lesley A. BAKER. 'The Kelmscott Press : To what Purpose ?' Journal of the William Morris Society, vol. XII, n², Spring 1997, pp. 36-38 (p. 36).

70. Morris n'est d'ailleurs pas le seul poète à apprécier la beauté des livres. Swinburne (1837-1909) verra dans The Golden Legend 'the most superbly beautiful book that ever, I should think, came from any press'. A.C. SWINBURNE, Lettre à F.S. Ellis (18 octobre 1892), citée dans William S. 
PETERSON, The Kelmscott Press, p. 216. De même, W.B. YEATS (1865-1939) écrira à Cockerell (dans une lettre non datée, citée p. 257) à propos du Chaucer : 'To me it is the most beautiful of all printed books'.

71. Quelque dix ans après avoir ainsi contrarié Cobden-Sanderson, William Morris déclarera: 'Good paper and good binding naturally follow good printing'. " "Master Printer Morris" : A Visit to the Kelmscott Press'. Entretien avec un journaliste non identifié publié dans la Daily Chronicle, 22 February 1893, p. 2. Reproduit dans The Ideal Book, op. cit., pp. 95-98 (p. 96).

72. Robinson cite un passage d'une lettre de Burne-Jones (1833-1898) qui montre en même temps la répartition volontaire des tâches avec William Morris et une fois encore l'analogie avec l'architecture : 'Indeed, when the book is done... it will be a little like a pocket cathedral. My share in it is that of the carver of images at Amiens, and Morris' that of the Architect and Magister Lapicida'. Duncan ROBINSON, William Morris, Edward Burne-Jones and the Kelmscott Chaucer, London: Gordon Fraser, 1982, p. 36.

73. Ian BRADLEY, William Morris and his World, London: Thames \& Hudson, 1978, p. 110.

74. Paul MEIER consacre de nombreuses pages solidement documentées à la réfutation du « luddisme intellectuel » dont certains l'ont accusé dans La pensée utopique de William Morris, Paris : Éditions sociales, 1972, pp. 481-492.

75. Peterson parle par exemple de 'occasionally uneasy compromises with modern technology'. William S. PETERSON, The Kelmscott Press, p. 313. Notons cependant que dans News from Nowhere, le tisserand ne cache aucunement qu'il travaille à la machine : 'I only do the most mechanical kind of weaving... Then besides the weaving, I do a little with machine printing and composing'. London: Penguin, (Revised) 1998, p. 58.

76. 'The workmen were paid higher than average wages for a forty-six-and-a-half-hour week'. Fiona McCARTHY, William Morris : A Life for our Time, p. 622.

77. Richard PEARSON, 'Review of William S. PETERSON, The Kelmscott Press : A History of William Morris's typographical Adventure.' Journal of the William Morris Society, vol. IX, n4, Spring 1992, pp. 38-41 (p. 40).

78. 'The true solace of Morris's last years lay not in politics, but in a new undertaking, the founding of the Kelmscott Press'. Christine POULSON, William Morris, London: Apple, 1989 (Réédition. The Art of William Morris, London: Quantum, 2004, p. 113).

79. Fiona McCARTHY, William Morris : A Life for our Time, p. 619

80. William S. PETERSON, The Kelmscott Press, p. 94.

81. Lettre à Cornell Price, juillet 1856. Norman KELVIN (ed.), The Collected Letters of William Morris, vol. II p. 28.

\section{RÉSUMÉS}

La dernière partie de la vie de William Morris est associée à l'image de la Kelmscott Press, qui peut être perçue comme une synthèse entre l'amour des livres qu'il a eu toute sa vie et la passion tout aussi longue qu'il a entretenue vis-à-vis des techniques de fabrication manuelles. Son sens de la beauté, issu de l'architecture médiévale, a dicté les choix esthétiques effectués pour les livres, qui à leur tour ont dicté les techniques retenues par la Kelmscott Press, caractérisées par le soin extrême apporté aux détails. Il a su combiner la grande connaissance qu'il avait des techniques traditionnelles du livre avec le savoir-faire de certains des plus grands artistes et 
artisans de son temps pour produire des ouvrages qui s'approchaient de très près de sa définition exigeante du « livre idéal ».

The last phase of William Morris's life is associated with the Kelmscott Press, which can be seen as providing a synthesis between his life-long love of books and his equally durable passion for manual methods of production. His sense of beauty, derived from medieval architecture, dictated the choices made for the appearance of the books, which in turn dictated the technical choices made by the Press, characterised by extreme attention to detail. He was able to combine his own extensive knowledge of traditional book techniques with the skills of some of the most prominent artists and craftsmen of his time to produce volumes which were the nearest thing to his exacting definition of 'the ideal book'.

\section{AUTEUR}

\section{ANTOINE CAPET}

Université de Rouen 